\title{
AD-0046 - Comorbidity of diabetes/prediabetes, obesity and arterial hypertension among women of Uzbek nationality
}

\author{
Rakhimova G. ${ }^{1}$; Alieva A. ${ }^{2}$; Ismailov S. ${ }^{3}$, \\ ${ }^{1}$ Tashkent Institute for Doctors' Postgraduate Education \\ ${ }^{2}$ Republican Specialized Scientific-Practical Medical Centre of Endocrinology \\ ${ }^{3}$ Tashkent Pediatric Medical Institute
}

Background and aim: Global epidemic of diabetes mellitus (DM) was the reason for discussion of this problem on the interparliamental level. But epidemic of other components of metabolic syndrome obesity and arterial hypertension is no less threatening. Aim: to study prevalence of comorbidity of diabetes/prediabetes, obesity and arterial hypertension among women of Uzbek nationality.

Materials and methods: we examined 1464 women of Uzbek nationality aged 35 and older, living in urban and rural areas of 3 regions of the Republic of Uzbekistan. Average age was $46 \pm 0.27$ years. Study included filling in of the questionnaire of DM risk based on FINDRISK, non-invasive measurement of blood pressure (BP) twice on both brachial arteries, oral glucose tolerance test with $75 \mathrm{~g}$ of glucose. DM and prediabetes were diagnosed according to IDF recommendations. Arterial hypertension (AH) was registered if both BP measurements were $140 / 90 \mathrm{~mm} \mathrm{Hg}$ and higher or examined women was prescribed and took any antihypertensive medicine. Obesity/ increased body weight were diagnosed if BMI were $\geq 30$ and $25 \mathrm{~kg} / \mathrm{m} 2$, correspondingly.
Comorbidity of arterial hypertension $(\mathrm{AH})$, overweight and obesity with carbohydrates metabolism disorders in Uzbek women

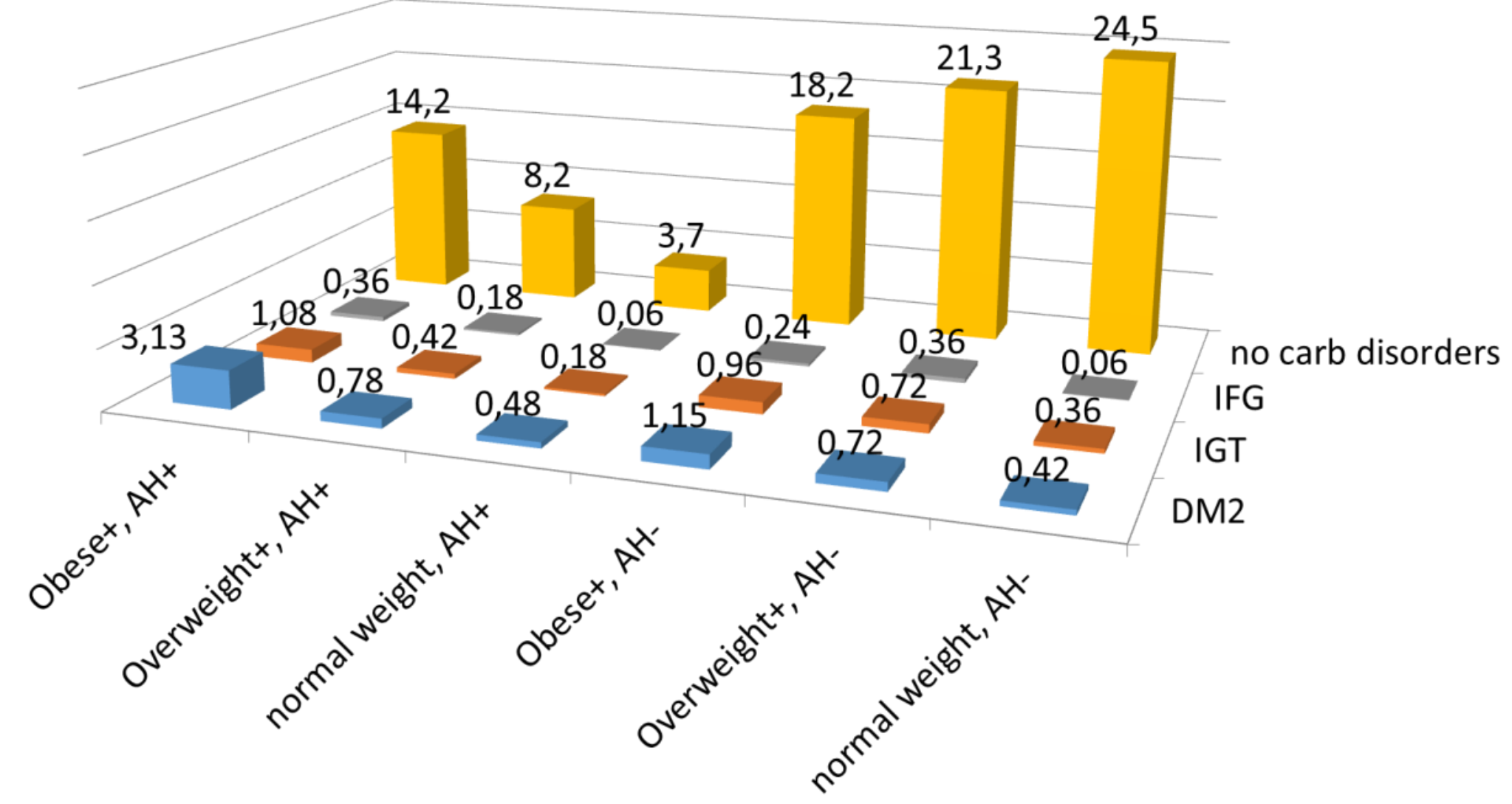

Results: $24.5 \%$ of examined women had no DM/prediabetes, nor increased body weight/ obesity, nor AH. DM/prediabetes were revealed in $13 \%$, of them only $7.7 \%$ had no increased body weight nor $\mathrm{AH} ; 6.7$ had $\mathrm{AH}, 31 \%$ - increased body weight/obesity; in 54\% of them carbohydrate metabolism disorders were concomitant with increased body weight/obesity and $\mathrm{AH}(7.2 \%$ of all studied women). It is a high prevalence of increased body weight/obesity among women without carbohydrate metabolism disorders: $67.6 \%$ were obese, including $22.9 \%$ who also had AH. $4.1 \%$ of examined women had isolated AH.

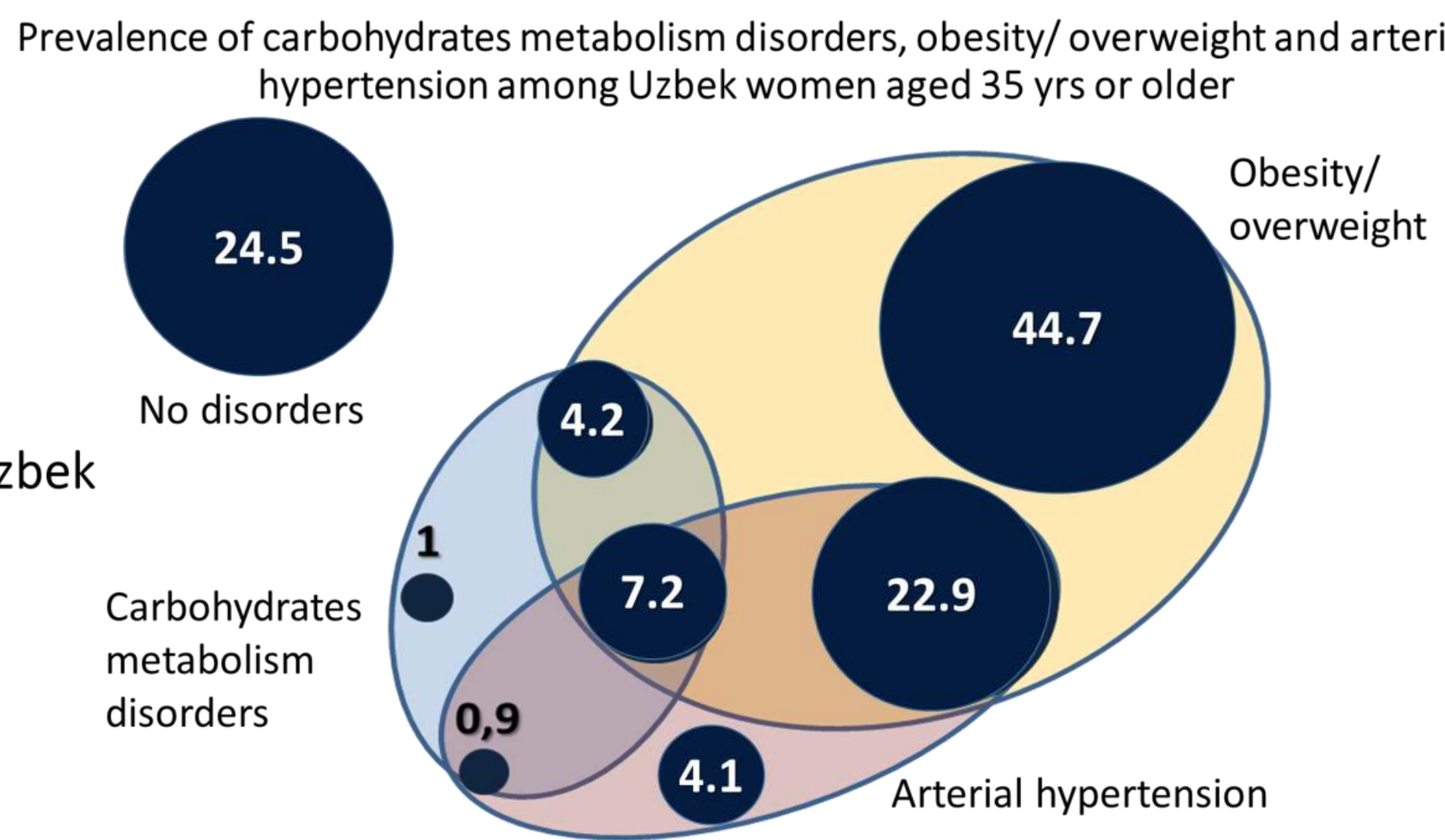

Conclusion: $7.2 \%$ of women of Uzbek nationality aged 35 and older had comorbidity of 3 dangerous conditions: diabetes/prediabetes, increased body weight/ obesity and arterial hypertension. High prevalence of increased body weight/ obesity claims more strict attention to this problem starting from the early years of life. 